\title{
The generation of neoplasia- enzymatic and emission
}

\section{Opinion}

A carcinogen or a mutagen is by characterization a natural or a synthetic, virus, infectious agent, radionuclide or radiation that amplifies and modulates carcinogenesis. Antecedent to which is the damage to the genome and the severance of cellular metabolism.

Cancer, by delineation, is a disease in which the normal cells do not undergo apoptosis at the same rate as mitosis. The deadly chemicals augment the exposure and conversion to the malignancy by altering the cellular viability and blemishing the DNA thus hampering with the biological processes and form the basis for disorderly, unchecked cellular proliferation. Extreme breakage of the DNA leads to programmed cell death, however, if the conduit is contaminated, the cell could change about to a neoplastic counterpart. The products with oncogenic potential are not instantly noxious and are often surreptitious.

Naturally occurring and dominant microbial inducers of malignancy are Aflatoxin B, a byproduct of fungus Aspergillus Flavus, found on stored nuts and grains. Viruses such as Hepatitis B, Human Papilloma Virus and Rous Sarcoma Virus are also generators of a neoplastic clone of cells. As are Infectious agents e.g. bacteria such as Helicobacter Pylori, helminthic parasites Opisthorchis viverrini, Clonorchis sinensis. Several irradiated items are carcinogenic due to the radiation such as gamma rays, alpha molecules etc. Non radioactive carcinoma inducers are synthetic compounds such as asbestos, dioxin, tobacco smoke etc, as are certain natural ingredients. ${ }^{2}$

Radionuclides are known to cause neoplastic transformation with emissions of alpha, beta, gamma or neutron radiation of strength. It is the genesis of ionization of the tissues, the carcinogenicity of which depends upon the type and degree and diffusion of radiation exposure.

Alpha radiation has a low infiltration and is non- hazardous in vitro but a health hazard when ingested or inhaled e.g. Thorotrast suspension (x-ray contrast) because of tissue confinement and incessant emission. Low-key ionizing radiation causes irrecoverable DNA catastrophe, replication and transcription deviations. Also elicit concomitant viral interactions to bring about premature aging and demise.

Low energy waves of the electromagnetic spectrum analogous to the radio waves, microwave, infrared radiation and visible light have inadequate energy to break the cellular chemical bonds. Neutron radiation yield inside the nuclear reactors are able to form secondary radiation due to nuclear transmutation. High energy radiation including Ultraviolet rays, sunlight, $\mathrm{X}$-rays, gamma radiation is also mutagenic.

Chemical compounds such as dioxins, dioxin like compounds, benzene, ketone, ethylene dibromide (EDB) and asbestos are encompassed in the checklist of mutgens. Industrial pollutants, tobacco smoke were catalogued in the early twentieth century as the causative factors with the yield of benzopyrene, tobacco specific nitrosamines, nitrosonornicotine and reactive aldehydes such as formaldehyde. Synthetical compounds in plastics, embalming fluid, vinyl chloride
Volume 4 Issue | - 2018

\author{
Anubha Bajaj \\ Laboratory A.B.Diagnostics, India
}

Correspondence: Anubha Bajaj, Laboratory A.B.Diagnostics, A-I, Ring Road, Rajouri Garden New Delhi I I0027, India, Email anubha.bajaj@gmail.com

Received: November 20, 2017 | Published: January 30, 2018

from PVC (polymerization of vinyl chloride) manufacturing are also implicated. Co carcinogens are alchemicals which do not initiate but contribute to the action of other mutagens (Figure 1).

\section{Mechanism of Carcinogenesis: Genotoxic Carcinogens}

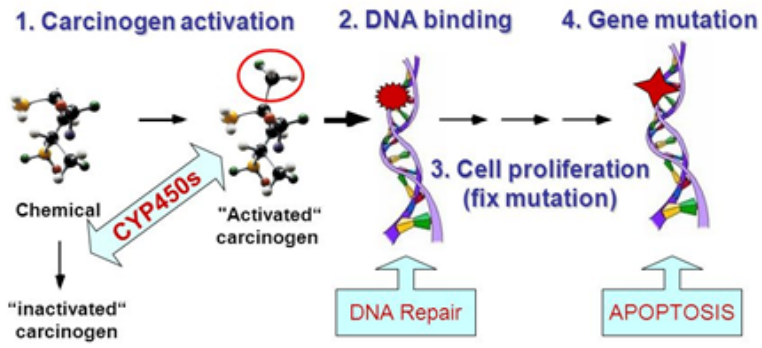

Figure I Mechanism of carcinogenesis: Genotoxic Carcinogens.

The corpus of tumorigenesis is the dispersal of radioactive and alchemical compounds that promote the formation of tumours. The carcinogenesis is due to the commencement and alteration of metabolic electrophilic reactants occurring in a natural setting. The compilation to a tumour is a multistage process is associated with the first stage of rapid and irreversible initiation caused primarily due to the covalent binding of the electrophilic derivatives of carcinogens leading to mutation of the cellular DNA.The second stage of promotion is a long occurring, circuitous course, the preceding changes in which are reversible. The hypercritical phase is epigenetic. The integrity of carcinogenesis is by both initiation and promotion, but the equation of the two may digress. ${ }^{3,4}$

After the carcinogenic ingression, there is a mechanism of its eradication through biotransformation, in a majority of the cases to make it water-soluble. However, in some instances the subordinate lethal component is converted to a supplementary lethal one. The DNA is nucleophilic and the soluble electrophiles are poisonous. Alkenes are enzymatically metamorphosed to procure electrophilic epoxide. DNA barrages the epoxide which is then contracted immutably. This contrivance is seen in carcinogenicity with tobacco, mustard gas, benzopyrene, aflatoxin, aromatic compounds etc. 


\section{Acknowledgements}

None.

\section{Conflict of interest}

Author declares that there is no conflict of interest.

\section{References}

1. Miller EC, Miller JA. Mechanism of Chemical Carcinogenesis. Cancer 1981;47(5 Suppl):1055-1064.
2. Chemical carcinogenesis and radiation carcinogenesis. The Medical Encyclopaedia. USA; 2003.

3. Oliveira PA, Colaço A, Chaves R, et al. Chemical Carcinogenesis. An Acad Bras Cienc. 2007;79(4):593-616.

4. http://slideplayer.com/slide/1468719/ 